\title{
The Value of Vague Ideas in the Development of the Periodic System of Chemical Elements.
}

\author{
Thomas Vogt \\ Departments of Chemistry \& Biochemistry and Philosophy \\ University of South Carolina
}

\begin{abstract}
The exploration of chemical periodicity over the past 250 years led to the development of the Periodic System of Elements and demonstrates the value of vague ideas that ignored early scientific anomalies and instead allowed for extended periods of normal science where new methodologies and concepts are developed. The basic chemical element provides this exploration with direction and explanation and has shown to be a central and historically adaptable concept for a theory of matter far from the reductionist frontier. This is explored in the histories of Prout's hypothesis, Döbereiner Triads, element inversions necessary when ordering chemical elements by atomic weights, and van den Broeck's ad-hoc proposal to switch to nuclear charges instead. The development of more accurate methods to determine atomic weights, Rayleigh and Ramsey's gas separation and analytical techniques, Moseley's x-ray spectroscopy to identify chemical elements, and more recent accelerator-based cold fusion methods to create new elements at the end of the Periodic Table point to the importance of methodological development complementing conceptual advances. I propose to frame the crossover from physics to chemistry not as a loss of accuracy and precision but as an increased application of vague concepts such as similarity which permit classification. This approach provides epistemic flexibility to adapt to scientific anomalies and the continued growth of chemical compound space and rejects the Procrustean philosophy of reductionist physics. Furthermore, it establishes chemistry with its explanatory and operational autonomy epitomized by the periodic system of elements as a gateway to other experimental sciences.

Keywords: History of Chemistry, Philosophy of Chemistry, Philosophy of Science, History of the Periodic System of Elements, Periodic Table of Elements, Prout's Hypothesis, Döbereiner Triads, Basic Chemical Element, Vagueness, Boundary Problems
\end{abstract}

\section{Introduction}

Scientific investigations are driven by the assumption that the physical world is at least partially intelligible. Complexities invite reductionism by assuming complex structures can be reduced to simpler ones and thereby provide explanations. The pursuit of a strong reductionist view was a paradigm in what was once called elementary particle and now is called high energy physics. This view is described by Steven Weinberg (2008): “All the properties of ordinary matter are what they are because of the principles of atomic and nuclear physics, which are what they are because of the rules of the Standard Model of elementary particles, which are what they are because...well we don't know, this is the reductionist frontier, which we are currently exploring." 
However, upon further investigations, building blocks of matter initially thought to be simple can turn out to be highly complex entities. Often quasiparticles can be more explanatory than the more elementary particles they are made up of. Cooper pairs and phonons are examples of quasiparticles with strong explanatory power for BCS superconductivity, where a conductor loses all electrical resistance below a critical temperature. However, those working at the reductionist frontier like Weinberg (2008) argue that this is not foundational work: "I think the single most important thing accomplished by the theory of Bardeen, Cooper, and Schrieffer (BCS) was to show that superconductivity is not part of the reductionist frontier. ...The great thing shown by Bardeen, Cooper, and Schrieffer was that no new particles or forces had to be introduced to understand superconductivity. According to a book on superconductivity that Leon Cooper showed me, many physicists were even disappointed that "superconductivity should, on the atomistic scale, be revealed as nothing more than a footling small interaction between electrons and lattice vibration".

Explanatory autonomy does not require chemistry or condensed matter physics to operate at the lowest level of the microphysical hierarchy, the reductionist frontier. Complexities emerge when units assemble and display unforeseen properties such as superconductivity. This challenges the strong constructionist claims of reductionism, because "the ability to reduce everything to simple fundamental laws does not imply the ability to start from these laws and reconstruct the universe" (Anderson 1972). This weak emergence stance decouples the laws of solid-state physics and chemistry from the 'reductionist frontier'. While Anderson (1972) refrained from the strong claim that higher-level laws in solid-state physics cannot in principle be derived from lower-level ones, he emphasizes that in practice we rely on references to empirically established phenomena ${ }^{1}$. This rejection of constructionist reductionism and embrace of weak emergence creates cultural and philosophical commonalities between scientists working at or across the blurred borders between chemistry and physics, i.e. in solid-state physics and solid-state chemistry - the history of this process is described in more detail by Martin (2018).

As we learn more and delve deeper into a science of matter our perception of a simple and coherent body of knowledge morphs and we become aware of the etiology and pathology of theories, models and concepts often hidden as approximations and not explicitly stated

\footnotetext{
${ }^{1}$ Superconductors are described using a phenomenological theory like BCS with few parameters (i.e. critical temperature $\mathrm{Tc}$ ) that are experimentally determined but cannot be calculated as materials properties from first principles.
} 
assumptions, some of which are metaphysical. One can follow the approach that approximations are a way to deal with complexities within a reductionist program and follow Dirac's proposition that "...the exact application of these laws leads to equations much too complicated to be soluble. It becomes desirable that approximate methods of applying quantum mechanics should be developed, which can lead to an explanation of the main features of complex atomic systems without too much computation (Dirac 1929)". Such an approach has been dismissed by developments in both solid-state physics and chemistry (Anderson 1972, Laughlin 2000a, 2000b, 2005). Today Dirac's approach has less and less impact on chemists beyond - for some - sustaining the myth of chemistry being reducible to physics. Proponents of strong reductionism lament the loss of precision and/or accuracy shifting from physics to chemistry. This crossover takes place in the condensed matter sciences, where a clear-cut disciplinary allocation is often not possible (Martin 2018). I would like to reframe this transition and ask what science gains when progressing from physics towards chemistry and other experimental sciences where we use distinct chemical concepts not reducible to or even conceptualized in physics such as valence, oxidation state, chemical bond, acidity, aromaticity, and electronegativity (Leach 2013). The latter is not derivable from first principles and not an observable but has been a very valuable idea in the theory of chemical bonding. These chemical concepts are introduced ad-hoc, as approximations, and conceptual discontinuities which make use of microphysical entities (i.e. electrons, protons, neutrons) and emergent quasiparticles (i.e. phonons, magnons, polarons), and well-established physical concepts (i.e. Coulombs Law, Thermodynamics) but address specific chemical phenomena. Chemists used concepts such as covalent bonding already around 1860 in a qualitative manner before Heitler and London (1927) were able to describe the simple hydrogen molecule quantitatively. Besides relying on qualitative concepts, chemistry operates highly inductive, even more abductive and thrives on heuristics expressed as rules, not laws, with often quite limited applicability. Quantum mechanics did not and cannot derive the periodic system of elements, nor the concepts of chemical bonding found in molecular and extended structures. The different lengths of the periods in PTE are not derived from ab-initio quantum mechanical calculations (Scerri 2012). Instead in a subfield of chemistry, quantum chemistry, we apply quantum mechanical tools to chemical systems (Schummer 1998), similar to how we approach superconducting materials. Chemistry develops concepts, theories, and models not exclusively by focusing on how accurate and precise they agree with experiments but by continuing to explore 
qualitative and vague ideas despite their initial shortcomings. In a short note over 80 years ago the physical chemist turned philosopher of science Michael Polanyi (1936) wrote: "The subject of chemical concepts, as opposed to physical ones, has always been fascinating to me because it shows the great value of inexact ideas...if at any time chemists would have been so ill-advised as to let themselves be frightened by physicists into abandoning all vague methods, and to restrict themselves to the fields where exact laws (or what are supposed to be such by the physicists) pertain, the development of chemistry, would at that moment have stopped dead, and its most valuable parts would have melted away in the rays of such foolish criticism."

Chemistry as a science defies the supremacy of reductionist physics and therefore needs a discipline-specific ontology. Metaphysical underpinnings of chemistry have been explored by Schummer (1996), Bensaude-Vincent (1998), van Brakel (2000), Cahn (2002), Harre (2005), Lombardi \& LaBarca (2005), and Scerri (2005). I will outline philosophical aspects of a central concept in chemistry, the periodic systems of elements, and sketch a brief and incomplete history of periodic systems and tables of the elements (PSE/PTE) ${ }^{2}$. I will present examples of how assumptions of simplicity drove the search for analytical and geometrical structure in the relationships between elements and provided constructive albeit vague ideas that allowed us to uncover some of the complexities of periodic systems. An important lesson of the history of PTE is that vague concepts such as the basic chemical element are adaptable to the epistemological complexity and inherent messiness of the chemical discovery process and might prove to be of use in other sciences. I will present evidence that vague ideas are productive vehicles of conceptual progress when developing a science of matter far from the reductionist frontier and use the wellestablished philosophical frameworks of Karl Popper (1963), Thomas S. Kuhn (1962), and Imre Lakatos (1980) throughout this text. I contrast Popper's falsification and Kuhn's historical approach which established that anomalies ${ }^{3}$ are often not acted upon but initially ignored and put aside for later reevaluation, thereby providing us long periods of 'normal science' in which we

\footnotetext{
${ }^{2}$ There are about 700 different periodic tables of elements (Mazurs 1974). Leal and Restrepo (2019) point to thousands of possible periodic tables of elements using different chemical classifications within the Mendeleevtype system. Therefore, I prefer to use the plural and have PTE understood as plural throughout the text when not specifically indicated by a qualifier. Periodic table of elements are $n$-dimensional mappings of periodic systems of elements (PSE), $n$ being mostly 2 or 3 . See further details in 2.1.

${ }^{3}$ Defining a Kuhnian anomaly and paradigm is often plagued by the fact that these terms are used for a wide range of cases and concepts and have vague definitions.
} 
engage in puzzle solving and methodological developments. Lakatosian research programs which are in contrast to Kuhn's paradigms heterogeneous and made up of a fundamental core and a protective belt where falsifications can occur without destroying the whole research program are useful philosophical frameworks in chemistry.

Allow me to make a few initial points about vagueness and its usefulness in chemistry. The problem of vagueness was introduced to philosophy by Eubulides of Miletus asking how many grains represent a heap. This Sorites paradox describes boundary cases where vague propositions do not allow one to decide if they are true or false. In modern logical terms, one would call vague propositions fuzzy. Vagueness and precision are characteristics occurring in representations and are well-established in linguistics. Representation has been addressed in chemistry by philosophizing chemists (Hoffmann \& Laszlo 1991, Hoffmann 1995, S. J. Weininger 1998) and historians (Klein 2003). As pointed out by Russell (1923), "Vagueness, clearly, is a matter of degree, depending upon the extent of possible differences between different systems represented by the same presentation. Accuracy, on the contrary, is an ideal limit." The meaning of chemical representations describing certain qualities is not precise but extends over a range making their meaning depending on the context. Many concepts in the physical and even more so in the chemical (and biological) sciences are vague and qualitative, relying on ranges of properties. Examples are strong and weak electrolytes, hard and soft acids and bases, concentrated and diluted solutions, or covalent and ionic bonds. In the latter case, we can assign a value to the range and state that a chemical bond has a certain degree of ionicity as derived for instance from a dipole moment measurement, but we can never define a sharp boundary between a covalent and ionic bond. ${ }^{4}$ Vagueness also relates to uncertainty and its different meanings, among them the lack of knowledge of an observer, experimental inaccuracies of measured quantities, and the distribution of an observable in an ensemble. Lack of knowledge of an observer is uncertainty regarding the microstates belonging to a macrostate such as the temperature of a system and led to the development of the concept of entropy in statistical physics. It also led to the discovery of the sensitivity of initial conditions and the evolution of chaotic systems and nondeterminism, one of the early chinks in the armor of reductionist physics. One can distinguish ontic and epistemological vagueness. At the beginning of the $20^{\text {th }}$ century, Heisenberg revealed uncertainty as an inherent

\footnotetext{
${ }^{4}$ This creates confusion teaching as we introduced different types of chemical bonds (i.e. metallic, ionic, covalent, polar covalent) but cannot provide clear boundaries for their existence.
} 
feature of molecules composed of atomic nuclei and electron densities with no sharp boundaries. A precise and complete description using complementary observables such as momentum and position is in principle not possible and does not depend on the quality of our measurement device

and measurement uncertainties. In chemistry, we sometimes find it helpful and explanatory to describe a molecule as a superposition of different structures when using the concept of resonance. Many are familiar with the two dominant resonance structures of benzene which alternate the location of carbon-carbon single and double bonds to account for a regular and planar hexagonal structure. This shows that often there is not a one-to-one but a one-to-many relationship between a chemical formula and its molecular structure - an example of a vague representation. As described in more detail in 2.2 the relationship between basic and simple chemical elements is an evolving one-to-many correspondence in the periodic system of elements. An important relationship between chemical elements in periodic systems defined by a vague concept is similarity, which helps us organize elements by their relation to other elements i.e. within a chemical group by using a range of different properties. For a more formal definition of similarity see appendix 1 in Restrepo (2019a). Similarity, a vague concept, tends to create boundary cases that resist classification since applied to an object $\mathrm{O}$ it can also be applied to any object $\mathrm{O}^{\prime}$ that differs only slightly from $\mathrm{O}$ - this results in Sorites-type paradoxes. We will see that the vague concept of similarity and order are the two equally important ingredients for periodic systems of elements.

\subsection{Chemical Periodicity, Periodic Systems, and Periodic Tables}

Using tables to represent classes of substances was first observed in Geoffrey's table des rapports from 1718 where columns listed classes of substances ranked in order of decreasing affinity to certain reactants. This "established the table as a paradigmatic organizational device" in chemistry as pointed out by Weininger (1998). However, reactions not material compositions were ranked. This tension between chemistry as a science of transformations and a science of matter is present throughout the history of PSE and has recently been revived in an attempt to "bring back chemistry to the system of chemical elements." (Restrepo 2019a, Leal \& Restrepo 2019). 
Chemical elements are related to each other in a system, the periodic system of elements (PSE), using both order (i.e. atomic weights, atomic numbers) and chemical similarities (i.e. formation of similar compounds). Historically order and similarities were derived from available chemical compounds (what we now call chemical compound space), initially by finding the smallest common weight of an element and similarity of the compositions, respectively. There are thousands of possible PTE because chemical elements can be ordered and classified according to many different properties. Placing chemical elements in mostly two- but also three- and higher dimensional systems creates spatial proximity which is often a proxy for certain physical similarities such as atomic weights, ionization potentials, or nowadays the number of protons present in the nucleus. This spatial closeness is also a proxy for the chemical similarity of reactivity due to common chemical valences or electronegativity and the formation of similar chemical compounds. However, proximity does not exclude significant differences of physical states and chemical properties of neighboring elements as exhibited by the horizontal neighbors $\mathrm{Au}$ and $\mathrm{Hg}$, the former a metal with a melting point of $1064^{\circ} \mathrm{C}$ and the latter a liquid metal at room temperature or liquid bromine's vertical two neighbors being chlorine gas and solid iodine at ambient conditions. The latter triad, chlorine, bromine, and iodine, are members of the chemical group of halogens sharing the same chemical valences ${ }^{5}$, which in early two-dimensional PTE were initially arranged horizontally and are now mostly arranged vertically. Elements of chemical groups form similar chemical compounds such as $\mathrm{HX}$ (for $\mathrm{X}=\mathrm{F}, \mathrm{Cl}, \mathrm{Br}$, and I) or $\mathrm{H}_{2} \mathrm{X}$ (for $\mathrm{X}=\mathrm{O}, \mathrm{S}, \mathrm{Se}$, and Te). Structural similarity in chemistry, a vague concept as defined above, is established by a common topology based on atom connectivity. PTE order and classify chemical elements. Initially, they were ordered by atomic weights and now atomic numbers. The classification is based on chemical similarities such as valences and combinations of other chemical similarities. This order-similarity requirement is a dual constraint for placing an element within a PTE. The history of PTE provides us many examples of competing orders and classifications expressed as boundary cases where different positions for elements such as hydrogen are given (Petrusevski 2018).

An example of a lesser-known 1-dimensional order, called the Mendeleev number $\left(\mathrm{M}_{\mathrm{n}}\right)$, was defined to have elements with similar chemical properties in close proximity as the number increases. It was originally proposed by Pettifor (1984) and modified by Villars (2004), who called

\footnotetext{
${ }^{5}$ Chemical valences are the number of hydrogen atoms that can combine with an element in a binary hydride, or twice the number of oxygen atoms combining with an element in its oxide or oxides.
} 
it the periodic number, and starts with Lithium $\left(\mathrm{M}_{\mathrm{n}}=1\right)$ and because it emphasizes chemical similarity, snakes through the vertical chemical groups placing hydrogen $\left(M_{n}=92\right)$ above the halogen group and $\mathrm{Be}\left(\mathrm{M}_{\mathrm{n}}=67\right)$ and $\mathrm{Mg}\left(\mathrm{M}_{\mathrm{n}}=68\right)$ above $\mathrm{Zn}, \mathrm{Cd}$, and $\mathrm{Hg}$. Placing hydrogen as the first element of the halogen group was suggested as it reacts with a metal and forms a hydride similar to halides. Villar's value of $\mathrm{M}_{\mathrm{n}}$ depends on where the hydrogen is located, either as a halogen or alkali metal. Initially, Pettifor found that binary compounds with the same structuretype can be found in proximity in a 2-dimensional map plotting the two element's $M_{n}$. These Pettifor maps were found empirically to be valid for many $A_{x} B_{y}$ compounds. Glawe et al (2016) derived a modified $\mathrm{M}_{\mathrm{n}}$ sequence based on the similarity of elements forming the same structures. The degrees of similarity between elements were calculated and a genetic algorithm was then used to determine an optimal sequence. $\mathrm{M}_{\mathrm{n}}$ is becoming increasingly important in phenomenological data-driven searches for new chemical compounds. More recently, Allahyari and Oganov (2020) revealed that a one-dimensional ordering using only atomic size and electronegativity allows the computation of a non-empirical $\mathrm{M}_{\mathrm{n}}$ and the derivation of a universal sequence of elements. Materials with similar properties such as hardness, magnetization, or enthalpy of formation cluster in chemical compound space, and the search for new materials is then simplified to exploring areas in the proximity of such cluster. This is of tremendous importance due to the vastness of chemical compound space, a higher dimensional space that contains all compositions and configurations (isomers) of chemical compounds. While huge, with an upper estimate of $10^{180}$ possible molecules and compounds (Lemonick 2020) it is populated very sparsely by functionalities. Clustering based on chemical similarity is an initial step to reduce chemical compound space and tackle the problem of mapping the desired functionality to existing chemical compounds from which to start the search for new compounds.

Chemical periodicity is the overarching concept that underlies the various PSE and PTE and states that chemical elements reveal similar chemical and physical properties in regular but varying intervals. These recurring trends can be qualitative trends such as the changes of atomic volume, ionization potential, or electronegativity. Chemical periodicity, as proposed by Mendeleev, describes the properties of simple substances, the constitution of their combinations, as well as the properties of the latter, as periodic functions of the atomic weights of the elements (Jensen 2002). Judged from today's perspective an "overstatement" (Restreop 2019b) it was an idea Mendeleev was reluctant to give up although it became clear to him that no rigorous analytical 
expression could be found and, as shall be explained below physical and chemical properties of simple elements were observed but basic elements listed in PSE and PTE.

The PSE based on applying both order and similarity to chemical elements has recently reemerged as a subject of mathematical investigations and can be described as ordered hypergraphs ${ }^{6}$ with similarity classes being hyper-edges. Many PTE are derived as mappings of these periodic systems into some lower-dimensional space (Leal \&Restrepo 2019). Mathematical tools used to study PSE involve number theory, information theory, order theory, set theory, topology, and even relating the cardinalities of the horizontal periods of PTE to an underlying Pythagorean structure involving triangular numbers (Restrepo 2007). Within the increasing use of machine learning in chemistry and materials science more and more phenomenological properties of chemical compounds are studied to extract heuristic rules without reference to first-principles quantum chemistry.

\subsection{Different Meanings of Element and Atom.}

Chemical elements have, to the chagrin of chemistry students, multiple meanings. One meaning refers to the basic element ${ }^{7}$ (Paneth 1962) and goes back to the Pre-Socratic elements earth, water, fire, and air, which were associated with four qualities (dry, cold, hot, wet) to describe the world. Elements were also associated with four Platonic solids: fire and the tetrahedron, earth and the cube, air and the octahedron, water and the icosahedron. As the concept of elements evolved, geometry was applied in an attempt to organize them and unearth an underlying structure. A $5^{\text {th }}$ Platonic solid of this 'elemental geometry', the dodecahedron, was assigned the function of a 'heavenly glue'. Aristotle also added a fifth element, an ether, which he posited the heavens were made of ${ }^{8}$. Plato and Aristotle rejected Democritus' speculative atomism whose metaphysical origin was in response to Parmenides' monism, which claimed 'Being' needed to be characterized by unity, permanence, indivisibility, and immobility. Pre-Socratic atomists agreed with the first three characteristics but rejected immobility and introduced atoms and voids to allow for change

\footnotetext{
${ }^{6} \mathrm{~A}$ hypergraph is a generalization of a graph in which an edge can join any number of vertices. In an ordinary graph an edge connects two vertices.

${ }^{7}$ Basic elements are also called abstract, metaphysical or transcendental elements. I will use the term basic here.

${ }^{8}$ The concept of an ether has a long history stretching from Plato and Aristotle over Descartes's theory of gravity to the many Michelson-Morley experiments searching for a medium for the propagation of electromagnetic waves. Walter Nernst proposed that radioactive atoms are created in an ether (Kragh 2012) and Mendeleev (Mendeleev 1904) claimed that there are two chemical elements, the element $X$ (Newtonium) and Y (Coronium) with lower atomic weights than hydrogen that make up the ether.
} 
by atomic mobility. The metaphysical element in Antiquity was a potentiality that gives rise to observable properties. Initially, it was a unitary view of matter where elements are immaterial qualities and always present. The proportions of the 4 elements of Antiquity are impressed on undifferentiated primordial matter and give substances their properties. The concept of the element in Antiquity was dominated by Aristotelian and not atomistic metaphysics. The basic element is a property bearer, an indestructible material ingredient of substances and unobservable. An important distinction is made between this basic and an observable simple element. The latter cannot be divided into constituents differing in kind. We maintain this position today but would add the qualifier "not separable by chemical means" as by using a mass spectrometer we can physically separate the different isotopes of chemical elements. The focus on observations and experiments encouraged by empiricism and early positivism in particular after 'Lavoisier's Chemical Revolution', amplified the importance of simple observable elements and pushed the previously important notion of basic elements into the background.

However, basic elements continued to be indestructible material ingredients of simple substances, an idea that can be followed back to Parmenides and later turned into Lavoisier's law of conservation of matter. In its atomistic version, this law was then advanced by Dalton in the $19^{\text {th }}$ century and the atoms of basic elements were associated with their corresponding atomic weights. In 1803 Dalton determined an element's atomic weight based on at least two independent series of compounds. (Rocke 2013 p149). Chemical atoms were used to explain the composition of chemical compounds. Dalton was confronted with the circularity of atomic weights and chemical formulas: if we know the chemical formula we can infer the atomic weights of the chemical elements from the combined weight and vice versa if we know the element's atomic weights we can derive the chemical formula. Initially, there were no independent experimental observations for neither and therefore one could set up arbitrary self-consistent systems of atomic weights and chemical formulas. As a way out of this dilemma, Dalton proposed a 'principle of simplicity' and the formula $\mathrm{HO}$ for water by using a speculative chemical theory: his atoms were rigid, stationary particles surrounded by 'atmospheres of caloric', which were understood as a medium of heat. At that time only water was known as a compound containing oxygen and hydrogen. Dalton postulated that only atoms of different chemical elements have chemical affinity and form molecules, while atoms of the same chemical element repel each other. Therefore, a molecule containing larger numbers of atoms of the same element will be less stable than one with 
a smaller number making an argument for HO. Only after the Karlsruhe Congress in 1860 did a consensus on atomic weights emerge after Cannizzaro promoted earlier ideas of Avogadro and Gay-Lussac. A shift of investigations to gaseous compounds and from atomic weights to volumes occurred and was aided by a theory of valence that provided a more coherent picture ${ }^{9}$.

Historically the physicist's atom was not the chemist's. The latter preceded Einstein's Ph.D. thesis on Brownian motion in 1905 and Perrin's subsequent experiments establishing physical molecules as well as Rutherford's model in 1911 (Rutherford 1911) by almost a century. Atomicity was still questioned in physics as late as 1897 when Ernst Mach stated emphatically after listening to a lecture by Ludwig Boltzmann in Vienna: "I do not believe that atoms exist" (Bächtold 2010). Many well-established chemists such as Wilhelm Ostwald and Marcellin Berthelot did not accept the notion of physical atoms. Instead, atoms and molecules were regarded as useful fictions for chemistry but not as real physical entities, and energy was claimed to be the fundamental reality. Berthelot's stated that atoms were not empirically warranted (Nye 1981). His stance against the need for physical atoms was further motivated by the fear chemistry might lose its explanatory and operational autonomy and be reduced to physics, a position upheld even after physical atomism was accepted and experimentally confirmed. This underlying fear of chemistry being reduced to physics was also voiced by Paneth (1962), who emphasized that "even if the character of chemistry should change essentially in the future owing to penetration by mathematico-physical methods, its history during the nineteenth century, in which it achieved such successes without mathematics, must never be ignored in its philosophic evaluation." This echoes Polanyi's statement quoted above (Polanyi 1936). By the time physical atomism was empirically established as a scientific paradigm, organic chemistry had already gone through a golden age of synthesis and formulated chemical transformations using molecules and atoms as 'useful fictions' (Ihde 1964). In his 1984 book (Rocke (1984, 320-325 \& 330-331) and a later article (Rocke 2013) Rocke makes the case that $19^{\text {th }}$-century chemistry had a theoretical foundation and pushes back against a narrow view of scientific theory articulated by some. The law of equivalent proportions in chemical compounds, an idea even the anti-atomists Berthelot and Ostwald accepted, brings about the necessity of the idea of chemical atoms in the nineteenth century (Rocke $(1984,320-325$ \& 330-331).

\footnotetext{
${ }^{9}$ For more details, see chapter 3 in Chang (2014) and Rocke (1984).
} 
We will see below that Mendeleev and Paneth ${ }^{10}$ emphasized the importance of basic elements used in PTE by their symbols such as $\mathrm{H}$ and $\mathrm{C}$, although they remain unobservable in contrast to the simple elements such as hydrogen gas, graphite, diamond, or graphene. There are many one-to-many relationships between basic and simple elements and more and more are being discovered by expanding physical parameter space (pressure, temperature), chemical synthesis (i.e. graphene, buckminsterfullerene $\mathrm{C}_{60}$ ), and exploring nanoparticles with different sizes and shapes. In Mendeleev's and other PTE, basic elements were ordered initially by their atomic weights and later by the number of protons in the atomic nuclei which are empirical observables that do not change during chemical reactions ${ }^{11}$ and are indestructible ingredients of all simple compounds. Basic elements are important concepts that continue to provide the exploration of the periodic system of elements with direction and explanation. It is even possible that towards the end of the periodic table the atomic number is no longer a well-behaved order parameter placing basic elements in groups with different chemical similarities.

\subsection{Prout's Hypothesis}

The first example of an important precursor of the PSE is Prout's hypothesis (Prout 1815, 1816), which advances ${ }^{12}$ a unitary view of matter, similar to what was suggested in Antiquity. It claimed that equivalent ${ }^{13}$ or atomic weights of elements are approximate integer multiples of the weight of hydrogen, the "protyle of the ancients" 14 and therefore there is only one state of matter manifesting itself in discrete and different assemblies. Prout's approach was to round off and approximate equivalent and atomic weights to the nearest integer and give hydrogen a value of 1 , assuming a representation of all element's mass in discrete units of the hydrogen mass.

\footnotetext{
${ }^{10}$ Friedrich Adolph Paneth's dual concept of element (1962) distinguished between the transcendental Grundstoff (basic substance) and einfacher Stoff (simple substance), which is the form in which the former manifests itself to our senses. The term transcendental was introduced as a nod to Kant (Ruthenberg 2009).

${ }^{11}$ We exclude radiochemical transmutations occurring in radiochemistry.

12 The English chemist Humphrey Davy already forwarded such an idea in 1808. In 1815 no atomic weight was known to even the nearest integer so the term Prout's speculation might be better. By the 1830 the discrepancies of the weight ratios from integer became larger and larger.

${ }^{13}$ The equivalent weight of an element is its gram atomic weight divided by its valence.

${ }^{14}$ This term refers to proto-hyle meaning 'first stuff' in Greek.
} 
This hypothesis motivated the research programs of many chemists among them Wolfgang Döbereiner and Alexandre de Chancourtois. The latter presented a 3-dimensional spiral as a PTE ordered by atomic weights in 1862, 7 years before Mendeleev's PTE. Furthermore, it spurred advances in measurement methodologies during a phase of 'normal science' which improved the accuracies of atomic weights ultimately leading to its rejection. This might appear very Popperian but the historical details as described by Scerri (2007a) are more complex: An early and strong rejection of Prout's hypothesis was presented by Jons Jacob Berzelius already in 1828. He objected to rounding off the values of atomic weights and wrote about Thomson, a supporter of Prout's hypotheses "...the greatest consideration which contemporaries can show to the author is to treat his work as if it never happened (Scerri 2007a)." However, Leopold Gmelin continued to use Prout's hypothesis and his practice of rounding off Berzelius' more accurate atomic weights. Certain ratios of atomic weights of elements to that of hydrogen are quite close to an integer value (i.e. O, C, N) but more and more non-integer boundary cases were found. After chlorine's atomic weight revealed a ratio to hydrogen's weight close to 35.5, Charles Marignac suggested in 1844 to reformulate Prout's hypothesis and normalize it to half the weight of hydrogen and thereby regain integer ratios. In response, Berzelius referred to Prout's hypothesis as 'Multiplenfieber' - German for 'a fever of multiples'. One can normalize to smaller and smaller fractions and in 1858 Dumas even suggested using $1 / 4$ of the value of hydrogen to rescue Prout's hypothesis. However, such adhoc amendments to include more boundary cases into an ordering scheme transformed a progressive research program into a stagnating and later even a degenerate one. Furthermore, Prout's hypothesis did not make any predictions expanding the scope of possible elements which could have further strengthened its claim. Despite this, even at the end of the $19^{\text {th }}$ century, Prout's hypothesis continued to motivate research by Lord Rayleigh that ultimately resulted in a strong challenge to Mendeleev's PTE as we will see below.

This brief historical summary shows that in chemistry we often follow a strong ontic bias for simple integer relationships - a bias we may call Pythagorean. These numerology-guided efforts to order the elements were later supplemented by geometry-inspired explorations. In 1867 Gustavus Hinrichs, who held on to Prout's hypothesis and was an ardent Platonist published a "chart of the elements" which has been credited to be the first circular PTE and incorporated boundary cases in Prout's classification among them chlorine (35.5) and aluminum (27.4) as integers with 71 and 55, respectively, by renormalizing appropriately (Zapffe 1969). In his 
'Atommechanik' Hinrichs related all elements to what he called the panatome. This arrangement created spokes of elements that contain many of what we now call chemical groups, he called them 'natural groups': The elements H-F-Cl-F-I, where hydrogen is placed in the halogen group are found in one spoke. These early geometrical attempts are the 2- and 3-dimensional precursors of n-dimensional graph theory as described by Restrepo (Restrepo 2019). There is a long history of searching for mathematical structures in the periodic system of elements and tables. Ordering and investigating the similarity of elements has and will continue to rely on mathematics. Statements about the non-mathematical nature of PSE and chemistry often stem from the aspiration to contrast vague and qualitative chemical concepts from highly mathematicized quantitative physical theories but are misguided attempts to "throw the baby out with the bathwater". Mathematical tools and concepts will always be used to explore PSE and extract PTE.

An initial bias for simplicity and integer values of ratios of physical parameters was very productive as the attempts to falsify Prout's hypothesis provided a sustained impetus to improve the methodologies to weigh atoms. This Galisonian aspect of tool and technique developments (Galison 1997) is an important characteristic of periods of normal science and complements the 'puzzle solving' activities Kuhn emphasizes. Furthermore, this process of improving experimental methods and agreeing upon a system of weights revealed an interesting characteristic as Rocke (2013) describes: "Between the 1820s and 1850s four systems of atomic weights and assumed molecular formulas competed for adherents in the European chemical world, those devised by Berzelius, Gay-Lussac and Dumas, Wollaston and Gmelin, and Gerhardt and Laurent. In 1858 Cannizzaro proposed a fifth one...In sum, despite its messy structure, the theory worked - and for quite a while it didn't seem to matter, too much, which version one used." (Rocke 2013 p149). That changed after the Karlsruhe congress in 1860 when valence and structural ideas based on the Cannizzaro weights resulted in a massive growth period in organic chemistry. This is an example of a period of normal science where we witness productive conceptual pluralism. It was not deemed necessary to immediately converge to one system of atomic weights to progress. And progress meant: "As the number of elemental atomic weights and presumed molecular formula expanded, as the numerical results were continually adjusted, and as gravimetric analyses steadily improved, a complex network of interdependent, inferential lines of argument, and implicit and explicit tests (both predictive and retrodictive) developed apace.” (Rocke 2013 p149). 
The Popperian notion that we readily discard falsified theories as anomalies appear is proven wrong by the almost eight decades during which Prout's hypothesis was still in play despite strong and growing evidence of anomalies. Instead, Kuhn's historical observation holds: Chemistry and other experimental sciences often initially ignore anomalies, cast them aside, don't accept them, or sustain the prevailing paradigm with ad-hoc hypotheses as was done in the case of chlorine and other elements which did not display integer atomic weight ratios with hydrogen. Ignoring boundary cases and postponing judgment on anomalies allows for extended periods of 'normal science'. There is temporary cloture on fundamental issues enabling methodological progress and the evolution of new tools - in Prout's case improving the measurement accuracies of atomic weights. There is a delicate balance between a radical openness and timely acceptance of anomalies advocated by Popper and Kuhn's cloture during periods of normal science where often a dogmatic approach will be established despite mounting evidence of anomalies. Popper's emphasis on a timelier response to anomalies would result in continuous and potentially inefficient reevaluations of the foundations of theories and models which might be abandoned too early. In an intriguing case study, Chang (2014) uses the phlogiston theory which was replaced during Lavoisier's 'Chemical Revolution' to make a case against abandoning theories or models prematurely and instead supports a pluralistic pursuit of explanation. Such an anti-monistic attitude is quite prevalent in chemistry and allows experimental methods, models, and concepts to pragmatically and productively coexist. Kuhn towards the end of his career developed ideas exploring an evolutionary philosophy of science (Marcum 2015) in which he discussed a new role for incommensurability, namely as an isolation mechanism that advances scientific practices by sequestering a community and giving it time and conceptual space in its 'evolutionary pond' to create what he called a lexicon

Kuhn (1977) in his work “Objectivity, Value and Theory Choice” lists simplicity, accuracy, consistency, scope, and fecundity as epistemic values for theory choice. Prout's hypothesis had an appealing simplicity but ultimately lacked the accuracy to account for the significant deviations from integer atomic weight ratios due to the improved accuracy of atomic weights. The atomic weight ratios resulted in boundary cases which could only be addressed by ad-hoc adjustments of the normalization and ultimately the realization that the atomic weights of elements are incommensurate with a unitary theory of matter. The hypothesis was not very fecund as it did not predict new elements to be discovered or connect with other theories. 


\subsection{Döbereiner Triads}

A Döbereiner triad (Döbereiner 1829) is a group of three chemical elements we would now find as members of a chemical group in $\mathrm{PTE}^{15}$. The atomic weight of the middle element is close to the average of the two others. This again points to a simple and approximate numerical relationship devoid of any initial explanatory theory at that time - a Pythagorean approach. Again, as in the case of Prout's hypothesis discussed above, the accuracy of the atomic weights of chemical elements was key to establishing or rejecting a triad. As triads occur in many chemical groups they also have similar chemical properties which makes them an important precursor of a PSE. In some triads such as $\mathrm{Cl}, \mathrm{Br}$, and I the average value of the mean of the atomic weights of $\mathrm{Cl}$ and I came very close to the atomic weight of Br. It was realized much later that the existence of Döbereiner triads was a very strong indication of chemical periodicity in PTE. Triads were revived and turned out to be very important: Nowadays Döbereiner triads are exact when we use atomic numbers instead of atomic weights and we understand that the inaccuracies encountered when using weights were due to the presence of different isotopes of chemical elements. In hindsight we can now explain why about half of the elements within a chemical group form a triad: if we arrange the PTE in its long-form we observe that the length of the periods (the number of chemical elements arranged horizontally in modern PTE) after the first one repeats once $(2,8,8$, $18,18,32,32)$ and therefore about $50 \%$ of the chemical elements have an element separated by the same number of elements before and after its position. Ignoring that the arithmetic means of the atomic weights in triads were only approximate and the fact that not all chemical elements were members of triads turned out to be productive. The underlying assumption that proved to be correct was that there must be some organization beyond the ordering of chemical elements based on atomic weights, that would only result in a one-dimensional string of chemical elements devoid of chemical information ${ }^{16}$. Exact triads based on atomic numbers are strong proxies for chemical periodicity introduced by chemical similarity and are still being discussed today: there is a triad $\mathrm{Y}, \mathrm{Lu}, \mathrm{Lr}$ that is currently being used as evidence in efforts to decide which elements should be

\footnotetext{
${ }^{15}$ Triads of chemical compounds such as the oxides $\mathrm{CaO}$, SrO and $\mathrm{BaO}$ were also found and therefore Döbereiner triads also reflect chemical similarity.

16 This does not imply that one-dimensional sequences of atoms cannot contain chemical similarities as Mendeleev numbers show.
} 
listed in group 3 (Scerri 2015). Scerri (Scerri 2012) correctly dispels the notion that electronic configurations of elements settle the position of elements in the $\mathrm{PTE}^{17}$ and proposes instead the maximization of triads of elements. The fact that initially only four elemental triads were found by Döbereiner did not prevent further developments by Gmelin, Dumas, and Lenssen, who in 1857 arranged the 58 known elements into 20 triads and explored relationships between triads (Scerri 2007e). The original triads were a progressive research program that hinted at structure in PTE which we now know is due to chemical similarities. Over almost 200 years the research program on atomic triads has metamorphosed from an initial Pythagorean attempt to order elements based on atomic weights to a concept that is now at the heart of chemical periodicity based on atomic numbers. This intriguing aspect of Döbereiner triads, the retrospective substantiation of chemical periodicity can also be understood as a discovery that initially could not be contextualized with concepts and theories available at its time - a premature discovery as Stent (Stent 1972) would call it. We further touch on this concept in section 2.6.

\subsection{Chemical Periodicity and Mendeleev}

One might think that the work on triads would inform and direct future work on PSE but surprisingly, Mendeleev did not take into consideration any prior work on triads for his PTE. The scientific discovery process in chemistry is highly complex and often does not follow an internal logic. An ahistorical and often heroic interpretation does not do justice to important details. The play "Oxygen" by Carl Djerassi and Roald Hoffmann (Djerassi \& Hoffmann 2001) uses the backdrop of a fictive Retro-Nobel Prize for the discovery of oxygen to argue that Scheele was the first to make it in the lab, Priestley the first to publish and Lavoisier understood the implications of its discovery for chemistry. Whiggish history credits exclusively Lavoisier and created the heroic myth of 'Lavoisier's Chemical Revolution'. The evolution of the Mendeleev-type PTE is no different. While heroic history in textbooks focuses on Mendeleev it is well established that, similar to Lavoisier's role during the discovery of oxygen, he was not the first to present an atomic weight-based PTE nor the first to publish, but understood its implications better and promoted his approach much more vigorously. Alexandre de Chancourtois in 1862, John Newlands in 1863,

\footnotetext{
${ }^{17}$ The Madelung Aufbau principle is valid up to $\mathrm{Ca}(\mathrm{Z}=20)$. Attempts to expand this heuristic rule to elements with higher $\mathbf{Z}$ has created wrong statements about an apparent energetic stability of full or half filled $d$-shells which unfortunately the majority of freshman chemistry books contain. Those that advance in chemistry are then required to 'unlearn' these myths.
} 
William Odling in 1864 and Lothar Meyer in 1864, Gustavus Hinrich in 1867 all preceded Dmitry Mendeleev's initial PTE published in 1869. Lothar Meyer's first PTE had 28 chemical elements organized into six chemical groups characterized by their valence and was published 5 years before Mendeleev's. It had two features Mendeleev is often exclusively given credit for: he left a gap for an element he predicted would be found subsequently and reversed the order of tellurium and iodine breaking with the atomic weight ordering and adhering to the chemical similarity based on the concept of valence, which was already well developed and mandated this positional switch from a chemical point of view. ${ }^{18}$ It is noteworthy that as a system based on ordering and chemical similarity of chemical elements according to their atomic weights is presented, there is a confirmed anomaly already from the onset, namely the tellurium/iodine ordering, that again represents a boundary case for order and similarity. In contrast to anomalies of Prout's hypothesis, which was challenged early on by other scientists, the tellurium/iodine switch was made by Odling, Mendeleev, Meyer, Newlands, and Hinrichs, and no PTE I am aware of was ever seriously challenged because it contained this or other element inversions. It appears that there was what one could call a tacit agreement among scientists that one was aware of this issue but agreed to continue within the framework of ordering by atomic weights despite having to acknowledge element inversions where chemical similarity based on valence superseded the physical property of atomic weight. We should think of the chemical elements in a Mendeleev-type PTE as positions on a horizontal string largely determined by increasing atomic weights and valences indicating where the 'carriage return' occurs to return to the beginning of the next period. Remarkably this PTE also incorporates the Döbereiner triads although Mendeleev insisted he was not aware of them or any other efforts to formulate a PTE and does not mention any of the earlier attempts in his writings. The question if this is deliberate neglect or unawareness of prior work cannot be answered without new historical sources. What sets Mendeleev apart from others was the fact that based on his PTE he foresaw the existence of eight chemical elements, namely scandium, gallium, germanium, technetium, rhenium, polonium, francium, and protactinium. This fecundity made his PTE a very progressive research program as it predicts and not just orders and classifies the known chemical elements. While not as extensive as Mendeleev, Meyer also predicted the existence of germanium, which was subsequently discovered by Clemens Winkler in 1886. However, Meyer

\footnotetext{
18 The same positional switch had already been done by William Odling in 1864 and most other PTE also had these two elements switched.
} 
did not make as many predictions of new chemical elements as Mendeleev and not with the same conviction and accuracy regarding their physical and chemical properties. Mendeleev used interpolation to predict physical and chemical properties with astonishing accuracy. The discovery of gallium highlights this: de Boisbaudran discovered it in 1875 and Mendeleev argued that eka$\mathrm{Al}$ as he called this element in his PTE should have a higher density - he was right. A classification and ordering scheme that predicts the existence of new chemical elements, some of them like francium and technetium discovered as late as 1939, extends the scope of possible observations and gives this PTE an advantage over one with more modest claims like Meyer's. Scope and accuracy are two of the five epistemic values Kuhn suggests for theory choice (Kuhn 1977) and they explain why this PTE became so well established. However, predicting elements that are experimentally found outweighs predicting non-existent ones that turn out to be 'false positives'. Depending on how one counts, Mendeleev predicted the existence of 6 to 8 more elements in a later PTE that do not, and as we know now cannot exist (Scerri 2007b), including an element for ether, which appeared to be more a 'fashion statement' (Mendeleev 1904 and footnote 10).

Overall Mendeleev ignored early anomalies and never doubted chemical periodicity as an overarching approximate law-like concept - a productive and vague idea. His anti-positivistic attitude as noted by Kultgen (1958) and later by Scerri was based on the fact that "the predictions Mendeleev made were thus conceived of with the abstract elements in mind." (Scerri 2007c). These basic elements of Mendeleev are metaphysical entities associated with an observable atomic weight that remains invariant in chemical reactions.

\subsection{Unexpected Noble Gases}

The next anomaly for the PSE/PTE research program was just around the corner. An important area of chemistry was the 'chemistry of airs', or gases as we call them now. In the 1770ies Scheele and Priestley had established that air was $80 \%$ 'phlogisticated air', what we now call nitrogen, and $20 \%$ 'dephlogisticated air', oxygen. A premature discovery, as mentioned above concerning Döbereiner's triads, is defined by Stent (Stent 1972) as an observation that cannot be contextualized with any of the prevailing scientific theories and paradigms at the time of its discovery. It is a stranded observation that might be rediscovered within a different historical context. Such a premature discovery was made by Henry Cavendish (Cavendish 1785): a minute 
residue of 1/120 of the original volume of air was left after separating and determining the amount of oxygen and nitrogen. In the 1890ies Lord Rayleigh and William Ramsey (Rayleigh \& Ramsey 1896) reinvestigated this unusual observation. However, the work's original motivation was Lord Rayleigh's attempt to investigate Prout's now 80-year-old hypothesis. Chemical analysis had advanced a lot and he found the ratio of oxygen's density to that of hydrogen to deviate significantly from the integer value of 16 . He then moved on to determine the density of nitrogen and after two years of work found that the density of nitrogen extracted from air and nitrogen made by chemical means differed by about $0.5 \%$. In his 1892 paper in Nature, he wrote: "I am much puzzled by some recent results as to the density of nitrogen, and shall be obliged if any of your readers can offer suggestions as to the cause" (Rayleigh 1892). When William Ramsey, a chemist from University College in London heard of Rayleigh's experiment he suggested that a yet undetected gas could be present. Rayleigh also learned of Cavendish's experiment 100 years ago. In a tour-de-force of analytical chemistry, they found and one might say 'rediscovered' a new chemical element, Argon. In their paper, they addressed the issue of premature discovery by Cavendish over a century before their work and graciously wrote: “Attempts to repeat Cavendish's experiments in Cavendish's manner have only increased the admiration with which we regard this wonderful investigation" (Rayleigh \& Ramsey 1896). But this discovery created an immediate crisis for Mendeleev's PTE: Argon gas was expected to be a diatomic gas molecule like other elements in the second period such as $\mathrm{N}_{2}, \mathrm{O}_{2}$, or $\mathrm{F}_{2}$. However, the ratio of the heat capacities at constant pressure and volume was too high. Diatomic molecules can absorb heat by changing their translation and interatomic vibration, monoatomic ones only by translation. With an expected atomic weight of 40 atomic mass units (amu) as a diatomic gas and therefore $20 \mathrm{amu}$ as an element, it would have fit nicely between $\mathrm{F}(19 \mathrm{amu})$ and $\mathrm{Na}(23 \mathrm{amu})$ albeit with no existing chemical group to join. However, as a monoatomic gas with $40 \mathrm{amu}$ it had, within the errors at that time, the same atomic weight as calcium and no chemical group to join. This created quite some excitement. Rayleigh \& Ramsey wrote: "If argon can be a single element then there is reason to doubt whether the periodic table of elements is complete" (Raleigh \& Ramsey 1896). A new chemical group, the noble gases, was added to the PTE. What could have become an ad-hoc proliferation of chemical groups quickly turned into the discovery of other members of this group. Argon was the first understood noble gas, however, helium had been observed already in 1868 in another pre-mature discovery in sunlight observed during a solar eclipse and named as a new 
element by Norman Lockyer ${ }^{19}$ after helios, Greek for the sun. It was subsequently found on Earth when outgassing the uranium-containing mineral, cleveite, by Ramsey in 1895 . The emanating gas had initially been erroneously characterized as nitrogen. William Crookes reconfirmed that this gas had the same spectroscopic lines as the ones observed earlier in sunlight by Lockyer. Further work and improvements in gas liquefaction led to Rayleigh's discoveries of neon, krypton, xenon, and Rutherford and Owen's discovery of radon - and more recently oganesson using an accelerator-based cold fusion technique in Dubna. This condensed complex history highlights the importance of premature discoveries in chemistry. Stent (Stent 1972) points to the observation that certain discoveries are incongruent with current knowledge and are dead-ends. There are many examples in the history of chemistry: Herman Staudinger first suggested the existence of longchain polymers in 1922, the prevailing paradigm was that they were aggregates of small molecules. He received the Nobel prize in chemistry in 1953 for his pioneering work that was derided for decades. Cole referred to these discoveries as delayed recognitions (Cole 1970) and citation analysis allows us nowadays to identify them as 'sleeping beauties'. Amongst them is a paper resulting in a recent Nobel Prize (Du 2016). A systematic bibliographic study using 22 million scientific papers by Ke et al (2015) showed that 'sleeping beauties' with long-dormant periods and fast citation growth after re-discovery are not as exceptional as previously thought and of the top 15 'sleeping beauties' 7 are in chemistry, followed by 5 in physics of which at least 3 are at the interface of physics and chemistry. Multidisciplinary physics and chemistry lead the top 20 disciplinary categories of 'sleeping beauties' identified in the Web of Science. Interestingly, many papers became important and even foundational in disciplines different from where they were originally published. There is no reasonable chain of interference that can connect Cavendish's 1785 paper to any prevailing paradigm at that time and it must therefore be classified as a premature discovery that was dormant for a century as a 'sleeping beauty'. In the same manner, in 1829 a Döbereiner triad could not be linked to what later became known as the long form of the PTE. The discovery of helium highlights that different scientific disciplines, in this case, astronomy and chemistry often operate and discover independently. The Herculean efforts by Raleigh \& Ramsey investigating the density differences between chemically made and atmospheric nitrogen highlights again the important role of methodological developments required

\footnotetext{
${ }^{19}$ Helium was detected during a solar eclipse in 1868 by the astronomers Georges Rayet, C. T. Haig, Norman R. Pogson and John Hershel and confirmed by Jules Janssen and Norman Lockyer who named it.
} 
to discover new chemical elements, something we will see also holds for the more recent discovery of superheavy elements.

\subsection{Atomic Numbers - At Last.}

In two remarkable papers in the magazine Nature, Anton van den Broek (van den Broek 1911 \& 1913), a scientific outsider trained in law and economics was the first to point to ordering chemical elements by their atomic number Z. Rutherford's experiment in 1911 (Rutherford 1911) used the scattering of alpha-particles on gold foils to establish the existence of a small and highly positively charged nucleus that carries most of the mass and prompted other scientists to look how other chemical elements scatter alpha-particles. A new tool was available to probe simple elements. Van den Broek analyzed data published by Hans Geiger and Ernest Mardsen (Geiger \& Mardsen 1913) from Rutherford's laboratory and found that the data agreed with Rutherford's theory provided nuclear charges are used and not atomic weights. As a scientific outsider and therefore unencumbered by prevailing paradigms or prior work van den Broek used a predominantly Pythagorean approach and ordered chemical elements by relating them to nuclear charges. This early part of the history of changing from atomic weights to atomic numbers is omitted in most chemistry books and courses and it is thanks to Scerri (2016) that we now have a detailed account of van den Broek's contributions.

In the same year as van den Broek published his second Nature paper, Moseley (1913) studied the frequencies of x-rays emitted from different metallic anodes hit by gamma rays and found an exact correlation between the square root of the frequencies of emitted x-rays and the atomic number $\mathrm{Z}$. If one plots the frequencies of emitted $\mathrm{x}$-rays as a function of atomic numbers one can now identify the previous anomalies and boundary cases found in Mendeleev-type PTE based on atomic weights: iodine and tellurium, argon and potassium as well as cobalt and nickel. If we order 6 chemical elements according to their increasing atomic masses the order is $\mathrm{K}$, Ar, $\mathrm{Ni}, \mathrm{Co}, \mathrm{I}$, and Te. If we take Moseley's atomic number $\mathrm{Z}$ and order them by increasing value, the order becomes Ar, K, Co, Ni, Te, and I - three pairs of neighboring chemical elements were inverted during half of a century's work ${ }^{20}$. 'Pair inversions' were important boundary problems

${ }^{20}$ All together there were 4 chemical inversions: $\mathrm{Te} / \mathrm{I}, \mathrm{Co} / \mathrm{Ni}, \mathrm{Ar} / \mathrm{K}$ and $\mathrm{Th} / \mathrm{Pa}$ 
that were resolved when transitioning to a new ordering - the atomic number. They were tolerated, without noticeable opposition, as anomalies during the continued exploration of Mendeleev-type PTE.

When switching to atomic numbers as an order parameter, vacancies at integer $\mathrm{Z}$ positions pointed to obviously missing elements: initially, Moseley's work indicated missing elements at $\mathrm{Z}=43$ (Tc), Z=61 (Pm), and Z=75 (Re). Later 4 more elements were added to this list: $\mathrm{Z}=72$ (Hf), $\mathrm{Z}=85$ (At), Z=87 (Fr), and Z=91 (Pa) (For more details see Scerri 2007 page 174-175). Altogether, seven elements were "missing" and their detailed histories are fascinating and illuminating case studies for the messiness of the discovery process (Scerri 2013). Ordering by atomic numbers no longer allowed 'false positive' claims of new elements but raises the new question of how high in atomic number we can go - an issue we touch on in 2.8 .

Lakatos' describes research programs as composed of a core and protective belt that contains positive and negative heuristics and connects the core to the world. Applying this to the PSE we would place the basic elements in the core. Each basic element is associated with an atomic number (i.e. $\mathrm{Z}=6$ for $\mathrm{C}$ ) in the protective belt, regardless of which simple element it is (graphite, diamond, graphene, or others) and how many neutrons are present in its atomic nucleus ${ }^{21}$. It is in the protective belt that falsification occurred: atomic weights no longer order the basic chemical elements in PTE and fail to uniquely determine simple elements as they can have isotope compositions different from their natural abundance. As a remarkable result of this, the same number of atoms of a simple element characterized by its number of protons can now have different weights. This is a radical change of the concept of a simple element: in the Mendeleev-type ordering by atomic weights samples with different weights but the same number of atoms could never be made up of the same chemical element. This was what Frederick Soddy pointed to in 1913: if atomic weight is the criterion for determining a basic element then there are positions in the PTE which are occupied by several 'elements', named isotopes, Greek for "the same place". What we witness here is "a radical redefinition of chemical elements away from Cannizzaro and Dalton's same mass' to Moseley, Paneth, Hevesy and Van den Broek’s 'same nuclear charge'

\footnotetext{
${ }^{21}$ Carbon has 15 isotopes from ${ }^{8} \mathrm{C}$ to ${ }^{22} \mathrm{C}$ with only ${ }^{14} \mathrm{C},{ }^{13} \mathrm{C}$ and ${ }^{12} \mathrm{C}$ occurring naturally and only the latter two are stable. The half-life times of most unstable isotopes are seconds and below with the exception of ${ }^{11} \mathrm{C}$ and ${ }^{14} \mathrm{C}$ with life times of about 20 minutes and 5730 years, respectively. Tying elemental existence to their half-life times becomes philosophically important in super heavy elements.
} 
qualifier" (Vogt 2017). There are debates if such a radical redefinition is a revolution in a Kuhnian sense (Brad Wray 2018) or not (Scerri 2018, 2021). An important consequence of this redefinition of a chemical element is the loss of the one-to-one relationship between types of atoms and chemical elements, which goes back to Dalton. For some like Fajans (see Kragh 2000 for details) this was not acceptable since important physical quantities such as entropy and specific heat vary with isotope composition. Paneth (1916) made the argument to stick with the basic elements in PTE and not use isotopes as they behave identically in chemical terms ${ }^{22}$. He stated unequivocally: "An element is a substance in which all atoms have the same nuclear charge". As a consequence of these developments, Kragh (2000) wrote: "The element and the periodic system are thus examples of conceptually robust chemical entities. Their histories indicate the force of the pragmatic chemical viewpoint and the value of retaining older theoretical notions, at least in a correspondence-like manner and up to a point. The reinterpretation of the element that occurred in the period kept the connection with the older concept through the principle of conservation of the elements in all chemical transformations." I agree with this important conclusion as both atomic mass and atomic number are invariants during chemical reactions when no nuclear transmutations take place. This invariance allows us to continue to explore chemical similarities of basic elements based on chemical compounds and relate them in PSE. The concept of basic elements is a "conceptually robust chemical entity" because it has been adaptable to boundary problems and anomalies and could be linked to different observables in the protective belt, first atomic weights and then atomic numbers. However, what defines an element nowadays is not only radically different from Mendeleev's days but can in some cases result in an incommensurability between the two definitions of an element based on either the same atomic mass or the same atomic number in the same way as we use the word mass both in Newtonian and Einsteinian physics but with quite different meanings: one as an absolute constant, the other dependent on the reference frame.

The history of PSE and PTE confirms Lakatos' view that all research programs will face anomalies and what matters is how they respond in either a progressive, temporarily stagnating, or degenerate way. Moseley's predictions are distinct from Mendeleev's as they ultimately pointed to where elements were missing and no longer allowed for 'false positives' which amounted to $50 \%$ of Mendeleev's predictions. Furthermore, the atomic number $\mathrm{Z}$ now became part of a new

\footnotetext{
${ }^{22}$ This is problematic for elements with low Z, in particular hydrogen with its isotopes deuterium and tritium where certainly the chemical reactivity is impacted and referred to as an 'isotope effect'.
} 
methodology with which claims of a new chemical element could be quickly falsified or confirmed. Georges Urbain claimed that he had discovered a new element with $\mathrm{Z}=72$ which we now call hafnium. A quick experiment in Oxford falsified this hypothesis. The PTE research program based on the chemical periodicity of basic elements is highly progressive as it has accommodated many anomalies, increased its scope by predictions, and strengthened chemistry's overall puzzle-solving capabilities by advancing methodologies and new characterization tools such as x-ray spectroscopy.

\subsection{Superheavy Elements - Trouble at the End?}

And so it should be no surprise that new challenges arose, this time with the discovery of superheavy elements (SHE) (Schädel 2015). These are transactinide elements starting with the atomic number $Z=104$ (rutherfordium) created artificially using cold fusion at accelerator facilities where for example a target like californium $(Z=98)$ is hit by a beam of calcium atoms $(Z=20)$ which gives us a few atoms of oganesson $(Z=118)$ currently the element with the highest atomic number ending the 7th period of PTE in the noble gas group.

A question that comes up regarding SHE is what does it mean when an element exists? The halflife times of SHE vary between about 28 hours for dubnium $(Z=106, N=268), 14$ minutes for seaborgium $(Z=106, N=269)$ to 1.9 seconds for flerovium $(Z=114, N=289)$, and 0.7 milliseconds for oganesson ( $\mathrm{Z}=118, \mathrm{~N}=294)$. Helge Kragh (2017) asks: "Can we truly say that the element oganesson exists when there is not a single atom of it in the Universe?" He points to a different ontology than for the elements of the first six periods, including short-lived radioactive elements. He suggests that we might better call such an element a 'potential element'. But where to draw the line? Another important distinction is that in many cases that when experimenting with SHE we have only a few atoms or possibly clusters of atoms but no longer elements as simple substances. Currently, chemical experiments have been done up to $Z=114$ (flerovium) which can be produced at a rate of about 5 atoms per day. The interactions of individual flerovium atoms with a gold surface indicated that it is the least reactive element of the carbon group but not as inert as the elements in the noble gas group as predicted by calculations (Yakushev 2014). This points to a second interesting question: possible changes in the chemistry of SHE as they might no longer fit in the chemical group they end up in due to their atomic number. This is at least partially due to relativistic effects which result in electron orbital contractions and expansions, shifting the 
electronic states that determine chemical reactivity and chemical valences. These effects can lead to different oxidation states, valences, ionic radii, and chemical bonding than observed for the lighter members of a chemical group. The chemical similarity and atomic number might no longer group similar elements vertically for higher SHE. New element inversions might need to be introduced. Recent calculations suggest that oganesson experiences a significant blurring of its electron shell structure due to relativistic effects (Jerabek 2018) which increases its polarizability and strengthens its interatomic van der Waals interactions suggesting a semiconducting solid at room temperature. This is in marked contrast to its 6th-period homolog radon, which is an insulating gas (Mewes 2019). Calculations by Pyykko (2011) predict possible element reversals in SHE at higher $Z$. This brings up another principle challenge to the periodic system. As Restrepo (2019b) states: "We have argued that order and similarity hold equal importance and one should not give preference to one over the other. If the vertical resemblance is not the rule and if the order by atomic number does not match the estimations of resemblance, has the time not come to reconsider the way of assessing resemblance and of overhauling atomic number as the order criterion?" While it is conceivable that we need to associate SHE with a different ordering criterion, their existence is currently based on their atomic number. The report of the IUAPC Transfermium group (IUPAC 2018) states: "Discovery of a chemical element is the experimental demonstration, beyond a reasonable doubt, of the existence of a nuclide with an atomic number $Z$ not identified before, existing for at least $10^{-14}$ s." Furthermore, in most cases, the element-specific chemical compound space based on chemical experiments will be quite small. For the near term, theoretical predictions will become important as their results can be included in PSE until experiments catch up.

The PSE continues to be an open research program as it is not even clear how many 'potential' chemical elements can be made. Based on extensive calculations chemical elements with atomic numbers larger than 172 are predicted to no longer be stable. This leaves us 54 new chemical elements to discover after Oganesson (Pyykkö 2011). Kragh (2019) points out that SHE can be thought about using the principle of plentitude, a metaphysical idea initially applied in biological sciences: "The extended plenitude formula thus becomes 'what can exist, either exists (or has existed or will exist) in nature or can be created" pointing to the different ontology of 
manufactured potential elements. An extended period of normal science might result in the development of new experimental and theoretical tools needed to enter the higher periods of PTE.

\section{Conclusions}

Jensen (1986), a philosophizing chemist noted the increased use of qualitative and descriptive concepts in chemistry alongside the causal mathematical relations prevalent in physics and attributed their occurrence to the increased complexity of observed phenomena and objects. Explanatory qualitative chemical and vague concepts such as the basic chemical elements, base, acid, ionic, and covalent bonds are classes whose behavior relates to a range of quantifiable properties. We have a one-to-many mapping of the representing to the represented system - a representation we introduced above as vague. This is in contrast to a one-to-one mapping of representing to the represented system for an explicit relationship where striving for the highest possible accuracy and precision of a measured property seeks to reduce its range. Jensen (1986) advanced a more general case for classification where the members have ranges of multiple properties. This classification matches the similarity relationships of elements in the periodic system of elements, a vague notion as noted above. A more rigorous argument about the periodic system of elements, understood as a mathematical set of related elements, is based on classification and order was made by Restrepo (2019): "They are neither a classification nor an ordering of elements, they are both! They are the interweaving of order and similarity relationships of the chemical elements." This takes into account that spatial proximity in PTE displays chemical similarity vertically in chemical groups and horizontally i.e. in ferrous metals (Fe, $\mathrm{Co}, \mathrm{Ni}, \mathrm{Pd}$ ), lanthanides, and actinides. For more detail see Restrepo (2019a and 2019b). There are many properties one can use to establish relationships between elements and therefore there can be thousands of different PTE. Using vague concepts such as similarity in science was in the past often called 'immature' or 'Linnaean' and deemed in need of reduction to physics. While this view has been challenged (Anderson 1972, Laughlin 2000a, 2000b) it unfortunately still exists as part of the Procrustean approach presented in introductory physics courses and popular science accounts. Instead of bemoaning a loss of accuracy and precision and being left with approximations and similarities, one should embrace the notion that "chemistry is the first science 
to deal in detail with a particular organizational aspect of matter, the first science to have species, the first science to have a natural history as well as a natural philosophy component, and consequently the first science to make pervasive use of class concepts" (Jensen, 1986). While chemistry is anchored in physicalism and relies on and extensively uses i.e. thermodynamics and quantum chemical concepts, it is the discovery of new chemical compounds that have and will continue to drive its progress. This search for new materials with targeted functionalities makes use of the proximity to regions identified in chemical compound space that provide starting compositions for explorations. Chemical discovery is more often not the result of a prediction but strongly rooted in a retrodictive rationalization of what happened. Jensen (1986) went on to describe sorting maps and their relevance to PTE. A Pettifor map deriving Mendeleev numbers as discussed above is an example of a sorting map. Meyer's famous atomic volume versus atomic weight plot is a sorting plot that reveals what Jensen calls "approximate symmetry in the form of an approximate periodicity". Functionalities localized in sorting maps lend themselves to interpolation and this is what Mendeleev and Meyer did when predicting Germanium and other elements. It was seen as a strength of the Mendeleev-type PTE and ultimately led to it being widely accepted. However, interpolation is fraught with errors: (i) not every property of elements is periodic or even vaguely periodic, (ii) we are measuring properties of simple substances but list basic elements in PTE and (iii) the recent wave of discoveries of allotropes in nanoscience, which are multiple realizations of basic elements complicates things further and point to more one-tomany relationships of the representing basic chemical element (i.e. C) to its many realizations as simple elements (graphite, diamond, graphene, $\mathrm{C}_{60}$, etc.). ${ }^{23}$ The latter also points to an open research program of deriving new PTE by applying similarity to a chemical compound space as a function of i.e. size, shape, pressure, temperature, and magnetic fields.

It is an interesting historical twist that both Prout's hypothesis and Döbereiner's triads can now be seen in a new light after ordering according to atomic numbers: The 'protyle' is the proton in the atomic nucleus and not the hydrogen atom, and the triads are exact when using nuclear charge. Lakatos called the revival of Prout's Hypothesis a "comeback" (Scerri 2007d), one could call it a transmogrification. These observations should caution us to attempt to guide and direct scientific work and judge models, concepts, and hypotheses as well as research programs too early

\footnotetext{
${ }^{23}$ Size dependent 'intrinsic' properties such as melting points of simple elements add a further dimension to PTE: 2.5 nanometer spheres of gold particles melt near $300^{\circ} \mathrm{C}$ whereas bulk gold melts at $1064^{\circ} \mathrm{C}$.
} 
and exclusively on their quantitative agreement to data. An important lesson is that a greater epistemic humility to sustain multiple and often opposing and vague concepts, models and theories can nurture research programs over extended periods. Such a scientific pluralism will provide epistemic flexibility to adapt to inevitable anomalies. Because of its distinct approach to theory and model building chemistry should be seen as a gateway to other experimental sciences such as biology, climate, geo- and environmental sciences, and engineering whose individual histories need to be studied and analyzed using existing and perhaps new philosophical frameworks. The diversity of the natural sciences should not be forced into a Procrustean philosophy of science developed mainly by exploring the history and philosophy of physics but instead seen as an opportunity to develop new discipline-specific philosophies.

The essence of a PSE can be boiled down to a system in which properties or combinations of properties of chemical compounds are ordered and classified according to their basic chemical elements. What anchors all explorations of a PSE is the basic element. While defined within a theory of matter by its atomic number, basic chemical elements have multiple realizations as simple elemental compounds and are indestructible material ingredients of simple substances in an evolving chemical compound space. The vastness of chemical compound space can be explored by using vague chemical similarities to point to local regions of interest in PSE for targeted functionalities. Increased use of machine-learning procedures will provide new predictions which can be updated as chemical compound space continues to grow. Likely, the PTE hanging on the walls of chemistry classrooms and laboratories will be replaced by virtual access to continuously updated chemical databases that will create user-defined PTE for specific chemical problems. A periodic system tailored for geoscience was introduced by Railsback (2003). Efforts in 'chemistry beyond the atom' led to initial attempts to devise a periodic system of supramolecular elements as an organizing and predictive tool of supramolecular matter dominated by noncovalent interactions (Schmidt \& Würthner 2020). These two examples illustrate the versatility and strength of PSE in subfields of materials science based on the order and similarity of chemical elements and molecular building blocks.

Periodic systems and tables might find use beyond chemistry, making use of vague concepts in other experimental sciences and exploring similarities of entities of interest such as perhaps genes or memes. The challenge will be to find an entity as robust, explanatory, and historically adaptable as basic chemical elements. If such systems can be devised and proven useful 
then chemistry would truly become the gateway to other experimental sciences. Polanyi (1936) ends his little note cited at the beginning with: "Chemistry, indeed, leads us far away from physics, (or let us say, that Physics appears when we look at Chemistry, so far remote from everything else in the world) that the description of chemical substances and the art of dealing with them lies quite near, by comparison, to the types of human behavior and the art of commanding human behavior." 


\section{References}

Z. Allahyari \& A. Oganov (2020). Non-empirical definition of the Mendeleev Numbers: Organizing the chemical space. J. Phys. Chem. C, 124, 23867 - 23878

P.W. Anderson (1972). More is different. Science, Volume 177, Number 4047, 393 - 396.

Aristotle De caelo, 302 a16

M Bächtold (2010). Saving Mach's view on atoms. Journal for General Philosophy of Science. Zeitschrift für allgemeine Wissenschaftstheorie Vol. 41, No. 1, Darwinism, Philosophy, and Experimental Biology, pp. 1-19.

B. Bensaude-Vincent (1998). Éloge du Mixte, Hachette, Paris.

H. Cavendish (1785). Experiment on Air. Phil. Trans. Of the Royal Society 75: 372-384.

R. M. Cahn (2002). Philosophische und historische Aspekte des Periodensystems der chemischen Elemente. Hyle Publication, Karlsruhe. www.hyle.org/publications/books/cahn/index.html. Accessed November 28, 2020.

Hasok Chang (2014). Is Water $\mathrm{H}_{2} \mathrm{O}$ ? Evidence, Realism and Pluralism. Springer, New York.

S. Cole 1970, Professional standing and the reception of scientific discoveries. Amer. J. Sociol. 76:286-306.

P.A.M. Dirac (1929). The Quantum Mechanics of Many-Electron Systems. Proceedings of the Royal Society of London A123, 714-733.

C. Djerassi, R. Hoffmann (2001). Oxygen. Wiley-VCH Verlag, Weinheim.

J. W. Döbereiner (1829). Versuch zu einer Gruppierung der elementaren Stoffe nach ihrer Analogie. Annalen der Physik und Chemie. 2nd series, 15 (2): 301-307.

J. Du, Y. Wu (2016). A bibliometric framework for identifying princes who wake up sleeping beauty in challenge-type scientific discoveries. Journal of Data and Information Science Vol 1 (1), 50-68.

P. Galison (1997). Image and logic: a material culture of microphysics. University of Chicago Press, Chicago.

Hans Geiger and Ernest Mardsen (1913). The laws of deflection of $\alpha$-Particles through Large Angles. Philosophical Magazine, 25, 604-628.

H. Glawe (2016), A. Sanna, E.K.U Gross, M. A. L. Marques. The optimal one dimensional periodic table: A modified Pettifor chemical scale from data mining. New. J. Phys. 18(9), 093011

R. Harré (2005). Chemical Kinds and Essences Revisited. Foundations of Chemistry, 7, 7 - 30.

W. Heitler, F. London (1927). Wechselwirkung neutraler Atome und homöopolare Binding nach der Quantenmechanik. Zeitschrift für Physik, 44, 455 - 472.

R. Hofffmann, P. Laszlo (1991). Representation in Chemistry. Angew. Chem. Int. Ed. Engl. 30, 1-16. 
Hoffmann, R.: 1995, The Same and Not the Same, Columbia University Press, New York.

J. Ihde (1964). The Development of Modern Chemistry. pp161 - 231. Dover Publications, New York.

IUPAC (2018). https://iupac.org/tag/transfermium-working-group/

W. B. Jensen (1986). Classification, symmetry and the periodic table, Comp. \& Math. with Appls., Vol 12B, Nos $1 / 2,487-510,1986)$

W. B. Jensen ed. (2002). Mendeleev on the Periodic Law: Selected Writings, 1869 - 1905. Dover, New York, $135-137$.

P. Jerabek, B. Schuetrumpf, P. Schwerdtfeger, and W. Nazarewicz (2018) Electron and Nucleon Localization Functions of Oganesson: Approaching the Thomas-Fermi Limit. Physical Review Letters 120, 053001.

J.H. Kultgen (1958). Philosophical conceptions in Mendeleev's principles of chemistry, Philosophy of Science, 25, 177-183.

H. Kragh (2000). Conceptual changes in chemistry: The notion of chemical elements, ca. 1900-1925. Studies in History and Philosophy of Physics 31: 435-450.

H. Kragh (2012). Walter Nernst: grandfather of dark energy? Astronomy \& Geophysics, Volume 53, Issue 1, February 2012, Pages 1.24-1.26.

H. Kragh (2017). On the Ontology of Superheavy Elements. Substantia, 1(2). https://doi.org/10.13128/Substantia-25 accessed November 28, 2020

H. Kragh (2019). Plentitude Philosophy and Chemical Elements. HYLE - International Journal for Philosophy of Chemistry, Vol. 25, No. 1, 1-20.

U. Klein (2003) Experiments, Models, Paper Tools: Cultures of Organic Chemistry in the Nineteenth Century. Stanford University Press, Stanford.

T. S. Kuhn (1962). The Structure of Scientific Revolutions. University of Chicago Press, Chicago.

T. S. Kuhn (1977) Objectivity, Value Judgment, and Theory Choice. Ch. 13, pp. 320-339 in The Essential Tension. University of Chicago Press, Chicago.

Imre Lakatos (1980) in John Worrall \& Gregory Currie, The Methodology of Scientific Research Programs: Volume 1: Philosophical Papers, Cambridge University Press, Cambridge.

R.B. Laughlin, D. Pines (2000a). The theory of everything. Proc. Natl Acad. Sci. U S A. 97 (1) 28-31.

R. B. Laughlin, D. Pines, J. Schmalian, B. P. Stojković, and P. Wolynes (2000b). The middle way. Proc. Natl Acad. Sci. U S A. 97(1): 32-37

R. B. Laughlin (2005). A Different Universe: Reinventing Physics from the Bottom Down, New York: Basic Books. 
Mark. R. Leach (2013). Concerning electronegativity as a basic elemental property and why the periodic table is usually represented in its medium form. Foundations of Chemistry Vol. 15, 13-29.

W. Leal, G. Restrepo (2019). Formal structure of periodic system of elements. Proc. Phil. Soc. A 475, 20180581.

S. Lemonick (2020). Exploring chemical space: Can Al takes us where no human has gone before? Chemical \& Engineering News, Vol 98 (13)

O. Lombardi, M. LaBarca (2005). The ontological autonomy of the chemical world. Foundations of Chemistry 7, $125-148$.

J. A. Marcum (2015). Thomas Kuhn's Revolutions. A Historical and Evolutionary Philosophy of Science? Chapter 6. Bloomsbury Academic, London, New Delhi, New York, Sydney.

J. D. Martin (2018). Solid State Insurrection. How the science of substance made American physics matter. University of Pittsburgh Press, Pittsburgh, Pa.

E. G. Mazurs (1974). Graphic Representations of the Periodic System During One Hundred Years. University Alabama Press: Alabama.

D. I. Mendeleev (1904). An attempt towards a chemical conception of the ether. Longmans, Green, and Co., London.

J-M Mewes, P. Jerabek, O. R. Smits, and P. Schwerdtfeger (2019). Oganesson is a semiconductor: on the relativistic band-gap narrowing in the heaviest noble gas solids. Angew. Chem. Int. Ed., 58, $14260-$ 14264.

H. G. J. Moseley (1913). The high-frequency spectra of the elements. Philosophical Magazine. 6th series. 26: 1024-1034.

B. W. Ninham, R. M. Pashley, P. Lo Nostro (2017). Surface forces: Changing concepts and complexity with dissolved gas, bubbles, salt and heat. Current Opinion in Colloid \& Interface Science 27, 25 - 32.

Mary Jo Nye (1981). Berthelot's anti-atomism: A matter of taste? Annals of Science 38(5), 585-590.

F. A. Paneth (1916). Über den Element- und Atombegriff in Chemie und Radiologie. Zeitschrift für Physikalische Chemie 91, 171-198.

F. A. Paneth (1962). The epistemological status of the chemical concept of an element. The British Journal of the Philosophy of Science 13, 1 - 14, $144-160$.

V. M. Petrusevski, J. Cvetkovic (2018). On the 'true position' of hydrogen in the Periodic Table. Foundations of Chemistry 20, $251-260$.

D.G. Pettifor (1984), Solid State Communications 51, 31.

M.Polanyi (1936), The value of vague ideas. The Philosophy of Science, Vol 13, 233-234. 
K. Popper (1963). Conjectures and Refutations. The Growth of Scientific Knowledge. Routledge and Kegan Paul, New York.

William Prout (1815). On the relation between the specific gravities of bodies in their gaseous state and the weights of their atoms. Annals of Philosophy, 6: 321-330.

William Prout (1816). Correction of a mistake in the essay on the relation between the specific gravities of bodies in their gaseous state and the weights of their atoms. Annals of Philosophy, 7: 111-13.

Pekka Pyykkö (2011). A suggested periodic table up to $Z \leq 172$, based on Dirac-Fock calculations on atoms and ions. Phys. Chem. Chem. Phys., 13, 161-168.

L. B. Railsback (2003). An earth scientist's periodic table of the elements and their ions. Geology, 31 (9): 737-740.

Lord Rayleigh (1892). Density of nitrogen. Nature 46, 512-513.

Lord Rayleigh and William Ramsay (1896). Argon, a new constituent of the atmosphere. Smithsonian Institution, Washington, D.C.

G. Restrepo, L. A. Pachon (2007). Mathematical aspects of the periodic law. Foundations of Chemistry 9 , 189-214.

G. Restrepo (2019a). Compounds bring back chemistry to the system of chemical elements. Substantia 3(2): suppl. 4: $115-124$.

G. Restrepo (2019b). Challenges for the Periodic Systems of Elements: Chemical, Historical and Mathematical Perspectives. Chem. Eur. J., 25, 15430 - 15440.

A. Rocke (1984). Chemical Atomism in the Nineteenth Century: From Dalton to Cannizzaro. Ohio State University Press, Columbus.

A. Rocke (2013). What did theory mean to nineteenth-century chemists? Foundations of Chemistry 15: $145-156$.

B. Russell (1923). Vagueness, The Australasian Journal of Psychology and Philosophy, 1:2, p. 90

K. Ruthenberg (2009). Paneth, Kant, and the philosophy of chemistry. Foundations of Chemistry 11: 79 91.

E. Rutherford (1911). The Scattering of $\alpha$ and $\beta$ Particles by Matter and the Structure of the Atom. Philosophical Magazine. Series 6, vol. 21, 669-688.

E. R. Scerri (2005). Some aspects of the metaphysics of chemistry and the nature of the elements. HyleInternational Journal of Philosophy of Chemistry, Vol 11, No 2, 127 - 145.

E. R. Scerri (2007a). The Periodic Table. Its Story and Its Significance. pages 38 - 42, Oxford University Press. 
E. R. Scerri (2007b). The Periodic Table. Its Story and Its Significance. Table 5.5 on page 142, Oxford University Press.

E. R. Scerri (2007c). The Periodic Table. Its Story and Its Significance. page 117, Oxford University Press.

E. R. Scerri (2007d). The Periodic Table. Its Story and Its Significance. page 182 and details in reference 49 of chapter 6, Oxford University Press.

E. R. Scerri (2007e). The Periodic Table. Its Story and Its Significance. 42 - 58, Oxford University Press.

E. R. Scerri (2012). What is an element? What is the periodic table? And what does quantum mechanics contribute to the question? Foundations of Chemistry 14: $69-81$.

E. R. Scerri (2013). A Tale of Seven Elements. Oxford University Press, New York.

E.R. Scerri (2015). The Constitution of Group 3 of the Periodic Table. IUPAC project number 2015-039-2200. https://iupac.org/projects/project-details/?project $\mathrm{nr}=2015-039-2-200$ accessed November 28, 2020.

E. R. Scerri (2016). A Tale of Seven Scientists and a New Philosophy of Science. Oxford University Press, New York, 41-62.

E. R. Scerri (2018). Letter to the Editor. Hyle- International Journal for Philosophy of Chemistry, 101-104.

E. R. Scerri (2021). Reassessing the notion of a Kuhnian Revolution. What Happened in Twentieth-Century Chemistry. Interpreting Kuhn. Critical essays. Edited by K. Brad Wray. Cambridge University Press.

M. Schädel 2015.Chemistry of the superheavy elements. Phil. Trans. R. Soc. A 373: 20140191.

H.-W. Schmidt and Frank Würthner (2020). A periodic system of supramolecular elements. Angew. Chem. Int. Ed., 59, 8766-8775.

J. Schummer (1996). Realismus und Chemie. Philosophische Untersuchungen der Wissenschaft von den Stoffen. Königshausen \& Neumann, Würzburg.

J. Schummer (1998). The Chemical Core of Chemistry. Hyle-International Journal for Philosophy of Chemistry, Vol. 4, No 2, $129-162$.

G, Stent (1972). Prematurity and Uniqueness in Scientific Discovery. Scientific American, Vol. 227, No. 6, 84-93.

A. van den Broek (1911). The Number of Possible Elements and Mendeléff's "Cubic" Periodic System. Nature Volume 87,78.

A. Van den Broek (1913). Intra-atomic Charge. Nature Volume 92, 372-373.

P. Villars, K. Cenzual, J. Daamsa, Y. Chen, S. Iwatac (2004). Binary, ternary and quaternary compound former/nonformer prediction via Mendeleev number. Journal of Alloys and Compounds 367, 167-175. 
T. Vogt (2017). Book review of Eric Scerri: A tale of seven scientists and a new philosophy of science. Hyle, International Journal for Philosophy of Chemistry 23: 107-109.

S. Weinberg (2008). From BCS to the LHC. Int.J. Mod.Phys.A 23 1627-1635.

S. J. Weininger (1998). Contemplating the Finger - Visuality and the Semiotics of Chemistry. HYLE - An International Journal for the Philosophy of Chemistry, Vol. 4, 3-27.

K. B. Wray (2018). The Atomic Number Revolution in Chemistry: A Kuhnian Analysis, Foundations of Chemistry 20: 209-217.

A. Yakushev, J. M. Gates, A. Türler, M. Schädel, C. E. Düllmann, D. Ackermann et al (2014). Superheavy Element Flerovium (Element 114) is a volatile metal. Inorg. Chem., 53, 1624-1629.

C. A. Zapffe (1969). Gustavus Hinrichs, Precursor of Mendeleev. Isis, Vol. 60, No. 4, 461-476 